\title{
Video Article \\ Visualizing Cell-to-cell Transfer of HIV using Fluorescent Clones of HIV and Live Confocal Microscopy
}

\author{
Benjamin Dale ${ }^{1}$, Gregory P. McNerney², Deanna L. Thompson ${ }^{2}$, Wolfgang Hübner ${ }^{3}$, Thomas Huser ${ }^{2}$, Benjamin K. Chen ${ }^{1}$ \\ ${ }^{1}$ Division of Infectious Diseases, Department of Medicine, Immunology Institute, Mount Sinai School of Medicine \\ ${ }^{2}$ NSF Center for Biophotonics, University of California, Davis \\ ${ }^{3}$ Structural and Computational Biology Unit, European Molecular Biology Laboratory
}

Correspondence to: Benjamin K. Chen at Ben.chen@mssm.edu

URL: https://www.jove.com/video/2061

DOI: doi:10.3791/2061

Keywords: Cellular Biology, Issue 44, HIV, GFP, live confocal imaging, virological synapse

Date Published: 10/7/2010

Citation: Dale, B., McNerney, G.P., Thompson, D.L., Hübner, W., Huser, T., Chen, B.K. Visualizing Cell-to-cell Transfer of HIV using Fluorescent Clones of HIV and Live Confocal Microscopy. J. Vis. Exp. (44), e2061, doi:10.3791/2061 (2010).

\section{Abstract}

By fusing the green fluorescent protein to their favorite proteins, biologists now have the ability to study living complex cellular processes using fluorescence video microscopy. To track the movements of the human immunodeficiency virus core protein during cell-to-cell transmission of human immunodeficiency virus, we have GFP-tagged the Gag protein in the context of an infectious molecular clone of HIV, called HIV GagiGFP. We study this viral clone using video confocal microscopy. In the following visualized experiment, we transfect a human T cell line with HIV Gag-iGFP, and we use fluorescently labeled uninfected CD4+ T cells to serve as target cells for the virus. Using the different fluorescent labels we can readily follow viral production and transport across intercellular structures called virological synapses. Simple gas permeable imaging chambers allow us to observe synapses with live confocal microscopy from minutes to days. These approaches can be used to track viral proteins as they move in from one cell to the next.

\section{Video Link}

The video component of this article can be found at https://www.jove.com/video/2061/

\section{Protocol}

This method was used in the research reported in Hübner et al. Science 323: 1743-1747 (2009).

\section{Overview}

Cell-to-cell spread of HIV was originally described using HIV infected Jurkat cells as donor cells and primary CD4+ T lymphocytes as acceptor cells ${ }^{1,2,3}$. In these studies, virus was detected in fixed cells using antibody staining. To track the transfer of virus from cell to cell in live cells, we exploit a recombinant, infectious molecular clone of HIV called HIV Gag-iGFP ${ }^{4}$. It carries the green fluorescent protein (GFP) inserted internally into the Gag protein between the MA and CA domains. This molecular clone of HIV retains infectivity without the need for helper virus. Virus particles made from this clone are stoichiometrically loaded with green fluorescent protein, providing a strong fluorescence signal to monitor viral assembly and transfer between cells. When used in conjunction with inert cell-tracking fluorescent dyes, recipient cells can be discriminated from input donor cells, and allowing one to visualize the transfer of HIV from an infected T cell to an uninfected T cell ${ }^{5}$. Virological synapse formation and transfer of virus from one cell to another can then be observed in living cells while they are cultured in a sealed, gas-permeable imaging chamber.

\section{(Day 1)}

\section{Preparation of HIV Gag-iGFP Transfected Jurkat T Cells}

1. Prepare the human CD4+ T cell line Jurkat (ATCC, Manassas, VA) by carefully maintaining the cells at a concentration between $2 \times 10^{5}$ and $8 \times 10^{5}$ cells $/ \mathrm{mL}$ in Jurkat culture media (RPMI 1640,10\% fetal bovine serum, 100 units $/ \mathrm{mL}$ penicillin, and $100 \mu \mathrm{g} / \mathrm{mL}$ streptomycin). Key to success: Culture of the Jurkat cells at concentrations in excess of $8 \times 10^{5} \mathrm{cells} / \mathrm{mL}$ will result in a reduction in transfection efficiency. Note: The transfection of HIV Gag-iGFP proviral DNA into T cells, as described below, results in the production of a replication-competent human immunodeficiency virus. As such, all procedures are to be undertaken only by trained laboratory personnel in certified biosafety level 2+ or biosafety level 3 (BL2+/BL3) tissue culture rooms. Work with infectious HIV in imaging facilities must be approved by your local institutional biosafety office. 
2. Transfect the Jurkats with the viral plasmid, HIV Gag-iGFP using the Amaxa nucleofection method (Lonza, Walkersville, MD). Pellet $5 \times 10^{6}$ cells by centrifugation for $10 \mathrm{~min}$ at $150 \mathrm{x}$. Aspirate supernatant and wash the cells in sterile phosphate buffered saline (PBS). Centrifuge the cells and resuspend the pellet to $97 \mu \mathrm{L}$ of pre-warmed, nucleofector solution $V$ containing the manufacturer's supplement. Add three $\mu \mathrm{L}$ of endotoxin free HIV Gag-iGFP plasmid $(1 \mu \mathrm{g} / \mu \mathrm{L})$ to the cell suspension and mix gently. Transfer the cell suspension to a cuvette and nucleofect using program S-18. Immediately transfer the cells to $3 \mathrm{~mL}$ of prewarmed Jurkat culture media without antibiotics.

3. To prepare CD4+ target cells for virological synapses, we obtain human peripheral blood mononuclear cells from buffy coats by using a standard Ficoll-Paque (GE Healthcare, Uppsala, Sweden) protocol. CD4+ T cells are then negatively selected using a magnetic bead isolation kit (Miltenyi Biotec, Auburn, CA). These may be cryogenically stored in liquid N2 in aliquots of $5 \times 10^{6}$ cells $/ 500 \mu \mathrm{L}$ of freezing media (90\% fetal bovine serum $/ 10 \%$ DMSO). Thawed primary CD4+ T cells are cultured in RPMI 10\% FBS, supplemented with 10 units/mL of IL-2.

(Day 2)

\section{Recovery of Live Cells from Jurkats Transfected with HIV Gag-iGFP and Staining of Target Cells with Fluorescent Dyes}

1. Allow the Jurkat cells to recover from the transfection overnight. Then remove the cellular debris by centrifugation over a ficoll hypaque density gradient material. Dispense $1.5 \mathrm{~mL}$ of Ficoll-Paque to the bottom of a $15 \mathrm{~mL}$ conical tube. Gently overlay this gradient material with the transfected Jurkats in a $5 \mathrm{~mL}$ volume of RPMI. Centrifuge the cells at $400 \mathrm{x} \mathrm{g}$ for $20 \mathrm{~min}$ at room temperature with the brake off. Carefully remove the cells from the media/Ficoll interface using a pipette. Transfer them to a new $15 \mathrm{~mL}$ conical tube. Dilute the cells with RMPI to a total volume of $15 \mathrm{~mL}$ and centrifuge at $300 \times \mathrm{g}$ for $10 \mathrm{~min}$. Resuspend the pellet in $3 \mathrm{~mL}$ of Jurkat culture media in a $2 \mathrm{~cm}$ well (6-well plate) and return the cells to the tissue culture incubator.

2. To distinguish target cells from donor cells in cell-cell transfer experiments we prelabel the CD4+ target cells with fluorescent dyes. Primary CD4+ $T$ cells are labeled the evening before use. We have had excellent success labeling $T$ cells with both CellTracker and CellTrace dyes (Invitrogen, Carlsbad, CA). While the dye concentration and incubation times must be optimized depending on the particular dye and cell type used, a representative protocol follows. Pellet $4 \times 10^{6}$ primary CD4+ T cells by spinning at $400 \times \mathrm{g}$ for 10 min. Wash the cells with 5 $\mathrm{mL}$ of PBS and resuspend in $2 \mathrm{~mL}$ of PBS. Add CellTracker Orange (CMTMR, Invitrogen, Carlsbad, CA) to a final concentration of $1.5 \mu \mathrm{M}$ and incubate at $37^{\circ} \mathrm{C}$ for $20 \mathrm{~min}$. Add $8 \mathrm{~mL}$ of complete Jurkat media and centrifuge at $400 \mathrm{xg}$ for $10 \mathrm{~min}$. Resuspend the cells in $3 \mathrm{~mL}$ of complete media supplemented with $10 \mathrm{units} / \mathrm{mL}$ of IL-2 and place in the tissue culture incubator overnight.

(Day 3)

\section{Monitoring Cell-to-cell Transfer of HIV Gag-iGFP using Live Cell Imaging}

We routinely use HIV Gag-iGFP transfected Jurkat cells 48 hours post-transfection. However, we have successfully used cells as early as 24 hours, and as late as 72 hours post-transfection.

1. Approximately 48 hours post-transfection, HIV Gag-iGFP expressing Jurkats are counted, washed with $\mathrm{CO}_{2}$-independent media (Invitrogen, Carlsbad, $\mathrm{CA})$, and resuspended in live cell imaging media $\left(\mathrm{CO}_{2}\right.$ independent media, $10 \%$ Fetal Bovine Serum, $100 \mathrm{U} / \mathrm{mL}$ penicillin, and 100 $\mu \mathrm{g} / \mathrm{mL}$ streptomycin, 10 units $/ \mathrm{mL} \mathrm{IL}-2)$ at a concentration of $1-3 \times 10^{7}$ cells $/ \mathrm{mL}$.

2. In a similar fashion, wash and resuspend labeled primary CD4+ T cells at a concentration of $1-3 \times 10^{7}$ cells $/ \mathrm{mL}$ in live cell imaging media.

3. Mix HIV Gag-iGFP-transfected Jurkats with primary CD4+ T cells at a 1:2 or 1:3 ratio and load into a tissue culture treated, gas permeable, microchamber (Ibidi, Verona, WI). For example, mix $20 \mu \mathrm{L}$ of HIV Gag-iGFP transfected Jurkats with $40 \mu \mathrm{L}$ of labeled primary CD4+ T cells. Load $50 \mu \mathrm{L}$ of this mixture into an Ibidi chamber and seal the chamber with plugs. Secure the plugs by wrapping the plug/lbidi chamber interface with parafilm

\section{Considerations for microscopy platforms: spinning disk confocal imaging}

After loading an Ibidi imaging chamber with cells, we immediately mount the device onto an inverted optical microscope (iX71 Olympus, Center Valley, PA). The Ibidi chamber should be transferred to the microscope only by BL2+-trained laboratory personnel wearing appropriate safety garments. Although live-cell imaging commonly uses expensive incubation chambers to maintain a stable $37^{\circ} \mathrm{C}$ environment, this was achieved using a simple and economical thermostatic heater (ASI 400, Nevtek, Williamsville, VA) along with a T-type thermocouple tip mounted next to the sample for proper temperature feedback. We have found using $\mathrm{CO}_{2}$-independent media allows us to maintain high cell viability while avoiding the use of a $\mathrm{CO}_{2}$ chamber. To buffer the platform against temperature fluctuations in the room and to block out background room light, a large black shroud is draped over the whole microscope/heater setup creating an insulated air pocket around the system. This greatly reduces temperature variation and associated temperature-related sample drift, but requires pre-heating for 30 minutes prior to mounting the sample.

\section{Data Acquisition, Transfer and Image Processing}

Images are taken using a 60x $1.42 \mathrm{NA}$ oil immersion objective (UPlanApo N, Olympus) as the high numerical aperture (NA) greatly improves resolution. To create 3D confocal images, scanning is performed by a spinning disk confocal unit (CSU-10, Yokagawa, Japan) that simultaneously uses $>800$ confocal spots to dramatically increase the scan rate. To compliment its speed, the CSU-10 is paired with a highly sensitive electron-multiplying charged-coupled device (EMCCD, iXon+897, Andor Technologies, Ireland) camera to allow fast, low light imaging which reduces both photobleaching and phototoxicity. The fluorescence light source is a multi-wavelength ArKr ion gas laser (Innova 70C, Coherent, Santa Clara) where the desired wavelength $(488 \mathrm{~nm})$ and laser power measured right before the microscope objective $(<1 \mathrm{~mW})$ are selected by an acousto-optic tunable filter (AOTF), (Andor Technologies). To further reduce photobleaching and extend total imaging time, the AOTF rapidly (microseconds) shuts off the laser beam every time the camera is inactive $(10-20 \mathrm{~ms})$ while the newly acquired image is delivered 
from the CCD (15-30 ms exposure) to the computer. The laser light is coupled into the spinning disk system using a 405/488/568/647 quad band dichroic mirror (Semrock, Rochester, NY). Fluorescence emission is filtered using a 525/50 bandpass filter (Semrock) inside a filter wheel (Ludl Electronic Products, Hawthorne, NY) mounted between the EMCCD camera and the confocal spinning disk unit. All hardware and acquisition, including a z-stage (Mad City Labs, Madison, WI), are controlled by the Andor iQ v1.8 software. To maximize the speed of acquisition at around 1.2-1.9 second 3D image stacks, we crop the recording region of the EMCCD's camera down to the cell-pair's immediate area. Furthermore, at the expense of some information in $z$, a large $z$-step between $0.45-0.75 \mu \mathrm{m}$ can be used to speed up acquisition. Imaging under these conditions can last up to 6 hours - using continuous 20-60 minute segments - with minimal photobleaching. Altogether, each data segment typically occupies $5-20 \mathrm{~Gb}$ of space as tens of thousands of images are taken. To facilitate the transport and analysis of the large data files, each acquisition set needs to be broken down and exported as $1 \mathrm{~Gb}$ TIFF image file segments using the Andor iQ v1.8 software. From here, many excellent software packages are available for image analysis, such as Metamorph, Volocity and ImageJ (we recommend its variation Fiji).

When two fluorescent markers are to be tracked, we simultaneously record both of them without slowing down acquisition by using a "split screen mode." Both markers are excited by two different ArKr laser lines at once (often 488 and $568 \mathrm{~nm}$ ). Inserted directly before the EMCCD camera is an image splitter (OptoSplit II, Cairn, Kent, UK) that separates the two different images. Each image is then independently filtered using fluorescence filters (Semrock) and projected side-by-side on the camera at once creating the "split-screen" effect. This would then require manual cropping and aligning of images later in image processing.

\section{Considerations for Image Processing and Data Analysis}

We typically perform image analysis with Volocity (PerkinElmer, Waltham, MA) on Macintosh computers (Apple, Inc). Spinning disk laser confocal microscopy images are first adjusted in their intensity to correct for photobleaching, then deconvolved with Volocity. Intensity measurements and tracking of Gag puncta is performed with the Volocity Quantification module. For image sets where the movement relative to a pre-formed synapse needs to be calculated, an automated tracking algorithm is employed which tracks the synaptic button throughout the entire sequence. Manual inspection of the regions of interest defined by the autotracking software must be performed to confirm that the software has correctly tracked the desired object. For objects where the contrast with the surrounding objects is too weak, manual tracking may be performed on a frame-by-frame basis. The Volocity software package allows the export of XYZ location, volume and integrated signal within the desired objects. The distance from the synapse and velocity of the tracked object are calculated by normalizing movements to the center of the synaptic button.

\section{Representative Results}

Within 10 to 15 minutes of mixing one should be able to visualize HIV Gag-iGFP expressing Jurkat cells adhering to primary CD4+ T cells. Imaging these conjugates, one can assess the movements of Gag puncta in infected cells as well as in target cells after synaspe. One may observe the movement of Gag-iGFP puncta into target cells soon after synapses are formed.

\section{Discussion}

We describe here a simple method for visualizing the transfer of HIV from cell-cell. Recent research from our laboratory and others' suggests that this may be the predominant mode of HIV spread between T cells. The method described here employs a recombinant form of HIV, called HIV Gag-iGFP, which carries a genetically encoded green fluorescent protein tag in the core structural protein, Gag. Because of the high levels of GFP produced in each virus particle, this replication-competent clone of HIV enables researchers to track individual virus particles in real time. This virus should be especially useful in studies concerning the assembly and cell-cell transfer of HIV.

The transfer of HIV by cell-cell transfer is highly efficient. During a three hour co-culture, we routine see virus transferred to $20-30 \%$ of primary $T$ cells as assessed by flow cytometry. Similar efficiencies are qualitatively seen during live cell imaging. Virally infected T cells begin forming synapses with uninfected primary T cells almost immediately after the initiation of co-culture. Interesting, not all primary $T$ cells are capable of interacting with HIV-infected Jurkat cells despite forced interactions with laser tweezers (unpublished observations, GM). HIV Gag-iGFP will undoubtedly be useful for determining T cell subsets that are most susceptible to cell-cell transfer, both in vitro and in vivo.

\section{Disclosures}

No conflicts of interest declared.

\section{Acknowledgements}

This review was supported by National Institutes of Health grants, Al074420-02, Burroughs Wellcome Investigator Award, Hirschl Career Scientist Awards to B.K.C. and by the NSF Center for Biophotonics Science and Technology, Cooperative Agreement PHY012099, and a UCD Health System Research Award to T.H. UCD CTSC NCRR ULRR024146.

\section{References}

1. Blanco, J. et al. High level of coreceptor-independent HIV transfer induced by contacts between primary CD4 T cells. J Biol Chem 279 , 51305-51314 (2004).

2. Sourisseau, M., Sol-Foulon, N., Porrot, F., Blanchet, F. \& Schwartz, O. Inefficient human immunodeficiency virus replication in mobile lymphocytes. J Virol 81, 1000-1012 (2007).

3. Jolly, C., Kashefi, K., Hollinshead, M. \& Sattentau, Q. J. HIV-1 cell to cell transfer across an Env-induced, actin-dependent synapse. J Exp Med 199, 283-293, (2004). 
4. Hubner, W. et al. Sequence of human immunodeficiency virus type 1 (HIV-1) Gag localization and oligomerization monitored with live confocal imaging of a replication-competent, fluorescently tagged HIV-1. J Virol 81, 12596-12607 (2007).

5. Hubner, W. et al. Quantitative 3D video microscopy of HIV transfer across T cell virological synapses. Science 323, 1743-1747 (2009). 